\title{
Quantum Spin Fluctuations as a Source of Long-Range Proximity Effects in Diffusive Ferromagnet-Superconductor Structures
}

\author{
A. Kadigrobov ${ }^{1,2}$, R. I. Shekhter ${ }^{1}$, and M. Jonson ${ }^{1}$, \\ ${ }^{1}$ Department of Applied Physics, Chalmers University of Technology and Göteborg University, SE-412 96 Göteborg, Sweden \\ ${ }^{2}$ B. I. Verkin Institute for Low Temperature Physics $\&$ Engineering, \\ National Academy of Science of Ukraine, 47 Lenin Ave., 310164 Kharkov, Ukraine
}

(22 December 2000)

We show that quantum spin fluctuations in inhomogeneous ferromagnets drastically affect the Andreev reflection of electrons and holes at a ferromagnet-superconductor interface. As a result a strong long-range proximity effect appears, associated with electron-hole spin triplet correlations and persisting on a lenght scale typical for non-magnetic materials, but anomalously large for ferromagnets.

In recent years much attention has been paid to normal conductor-superconductor $(\mathrm{N} / \mathrm{S})$ structures in which transport properties of the normal conductor are much modified in the vicinity of the superconductor (for a review, see paper 11]). The origin of this "proximity effect" has to do with correlations of normal-metal electrons and holes caused by Andreev reflection at the inteface with the superconductor. An important feature of such Andreev scattering, which converts electrons into holes and vice versa, is the selection rule that requires the energies of the electron and hole (as measured from the Fermi energy) - as well as their spin projections - to be equal in magnitude but opposite in sign. While the spin selection rule is irrelevant for the proximity effect in nonmagnetic normal materials, the energy selection results in a destructive interference between the electron and hole states corresponding to a decay of the electron-hole correlations at distances of order $L_{T}=\sqrt{\hbar D / k T}$ from the $\mathrm{N} / \mathrm{S}$ interface $(D$ is the diffusion constant of the normal conductor, $T$ is temperature).

In ferromagnetic materials electrons and holes acquire an additional exchange energy, which is sensitive to the direction of the spin. Hence the spin selection rule for Andreev reflection becomes relevant. As a result the proximity effect decays on the much smaller length scale $L_{I_{0}}=\sqrt{\hbar D / I_{0}}\left(I_{0}\right.$ is the exchange energy). Typically, $I_{0}$ exceeds $k_{B} T$ by several orders of magnitude, resulting in a drastic reduction of the proximity effect in magnetic materials as indeed observed in a number of measurements Such a shortening was indeed observed in a number of measurements (see Refs. [2 [5] and references therein).

Recently, new experiments [6] 8 revealed a large excess conductance of the F/S boundary, which was interpreted in terms of a long-range proximity effect in the ferromagnet. It was pointed out [7] that spin triplet fluctuations in the electron-hole correlations caused by the spin-orbit interaction and electron-impurity scattering [9] can not (by two orders of magnitude) explain the large effect ob- served in Refs. 6 . 8 .

The main message of our paper is that in magnetically inhomogeneous materials (such as multi-domain ferromagnets, inhomogeneous "cryptoferromagnetic" states imposed by the superconductor [10], F/S interfaces inducing electronic spin-flip processes [11 etc.), strong quantum fluctuations of the electron and hole spins make the proximity effect less sensitive to the spin selection rule that applies to Andreev reflections. As a result, a strong long-range, spin-triplet proximity effect in $\mathrm{F} / \mathrm{S}$ structures persists on a length scale typical for non-magnetic materials. We estimate the conductance of such an F/S structure to be of the same order of magnitude as the conductance measured in experiments [6] 8]. Additional experiments with intentionally introduced magnetic inhomogeneities are needed to check the predicted effect quantitatively.

An analytical solution for the proximity effect was obtained for the case when spin scattering occurs at distances shorter than the electronic mean free path $l_{0}$. This allows us to consider perfect Andreev reflection and spin scattering using the Eilenberger equation and to formulate proper boundary conditions for the complex scattering at magnetically inhomogeneous $\mathrm{F} / \mathrm{S}$ interface. These boundary conditions were used to solve the Usadel equation in the diffusive region of the ferromagnet and for calculating the excess conductance of the $\mathrm{F} / \mathrm{S}$ boundary. We show that a new type of superconducting ordering corresponding to spin triplet correlations is the source of the proximity effect at distances of order $L_{T} \gg L_{I_{0}}$. In order for an analytical solution to be feasible, we have considered the case of weak magnetic scattering. This is not a condition for the existance of the proposed longrange roximity effect, but it allows us to conveniently linearize the effective Usadel equation for the triplet components of the Green's function.

The Green's function of the problem, $\hat{\mathrm{g}}$, is an $8 \times 8$ matrix, which is the tensor product of the Keldysh $2 \times 2$ and the particle-hole $(\mathrm{Nambu}+\mathrm{spin}) 4 \times 4$ matrices. Hence

\footnotetext{
${ }^{1}$ One can show that the result expressed in terms of the spinflip amplitude does not depend on the ratio between $l_{0}$ and $L_{I_{0}}$
} 


$$
\hat{\mathbf{g}}=\left(\begin{array}{ll}
\hat{g}^{R} & \hat{g}^{K} \\
\hat{0} & \hat{g}^{A}
\end{array}\right)
$$

where $\hat{g}^{R, K, A}$ are retarded (R), Keldysh (K) and advanced (A) $4 \times 4$ matrix Green's functions which include both the singlet $\hat{g}_{\sigma,-\sigma}$ and triplet $\hat{g}_{\sigma, \sigma}$ components of the normal as well as anomalous Green's functions. The pairing potential $\hat{\boldsymbol{\Delta}}$ determining electronhole correlations, can be written in terms of Pauli matrices $\hat{\sigma}_{i}$ and the superconducting energy gap $\Delta: \hat{\boldsymbol{\Delta}}=$ $\hat{\sigma}_{0} \otimes \hat{\sigma}_{3} \otimes\left(\Delta^{*} \hat{\sigma}_{-}-\Delta \hat{\sigma}_{+}\right)$with $\hat{\sigma}_{ \pm}=\hat{\sigma}_{1} \pm \hat{\sigma}_{2}$. The Eilenberger equation [13] in the ballistic region $x \leq b$ (see Fig.1) is written as

$$
i v_{F} \mathbf{n} \frac{\partial}{\partial \mathbf{R}} \hat{\mathbf{g}}+\left[\epsilon \hat{\tau}_{3}+\hat{\boldsymbol{\Delta}}-\hat{\mathbf{h}}, \hat{\mathbf{g}}\right]=0,
$$

where $\epsilon$ is the energy measured from the Fermi level, $\mathbf{n}$ is a unity vector along the electron momentum, $\hat{\mathbf{h}}$ is the operator that describes the effect of the inhomogeneous magnetic moment $\vec{h}(x)=I_{0} \vec{e}(x)$ on the spins of electrons and holes $(\vec{e}(x)$ is a unit vector): $\hat{\mathbf{h}}=$ $\hat{\sigma}_{0} \otimes\left(h_{x} \hat{\sigma}_{1} \otimes \hat{\sigma}_{0}+h_{y} \hat{\sigma}_{2} \otimes \hat{\sigma}_{0}+h_{z} \hat{\sigma}_{3} \otimes \hat{\sigma}_{3}\right)$.

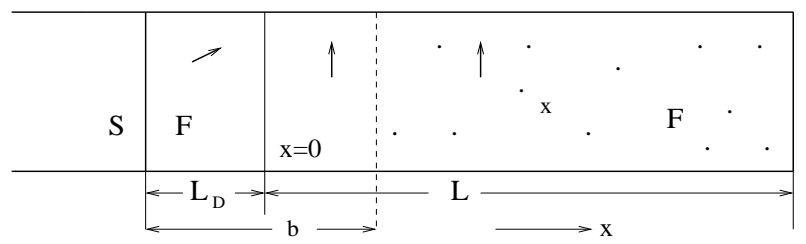

FIG. 1. Schematic view of an S/F structure with a domain wall at $x=0$, a distance $L_{D}$ from the $\mathrm{S} / \mathrm{F}$ interface. Impurity scattering is assumed to occur out of the ballistic region (to the right of the vertical dashed line).

Under the assumption that "spin splitting" due to magnetic scattering is small, one can find the solution of Eq.(2) in a ballistic region which includes a magnetic inhomogeneity (Cf. Fig.1). Outside the ballistic region, $x \gg l_{0}$, the Usadel equation [14] controls the proximity effect both for the singlet and the triplet components of the isotropic part of the matrix Green's function $\hat{\mathbf{G}}=\langle\hat{\mathbf{g}}\rangle(\langle\ldots\rangle$ denotes an average over the directions of electron/hole momenta). One finds that while the singlet components decay at distances of order $L_{I_{0}}$, both the normal and anomalous spin triplet components persist at much longer distances of order $x \approx L_{T}$. Using this fact one can linearize the problem with respect to the triplet components of the anomalous Green's function - the only ones that survive at distances much larger than $x \gg L_{I 0}$. As a result, one gets both the Usadel equation and its boundary conditions 2 as

${ }^{2}$ While deriving the boundary condition we used the fact

$$
\begin{gathered}
\hbar D \frac{d^{2} \Theta_{\sigma}}{d x^{2}}-2 i \epsilon \Theta_{\sigma}=0 \\
\left.\frac{d}{d x} \Theta_{\sigma}\right|_{x=0}=\sigma \frac{v_{F}}{D}\left|\left\langle\left|n_{x}\right| r_{s f}\right\rangle\right| .
\end{gathered}
$$

Here $r_{s f}$ is the magnetic spin-flip scattering amplitude, $\sigma= \pm 1$. In the above equations we have used the standard parametrization (see, e.g., Ref. [16]) of the triplet Green's functions for normal pairing $G_{\sigma, \sigma}^{R}, \bar{G}_{\sigma, \sigma}^{R}$ and anomalous pairing $F_{\sigma, \sigma}^{R}, \bar{F}_{\sigma, \sigma}^{R}$. It follows that

$$
\hat{G}_{\sigma, \sigma}^{R}=\left(\begin{array}{cc}
\cosh \left(\Theta_{\sigma}\right) & \sinh \left(\Theta_{\sigma}\right) \exp \left(i \chi_{\sigma}\right) \\
-\sinh \left(\Theta_{\sigma}\right) \exp \left(-i \chi_{\sigma}\right) & -\cosh \left(\Theta_{\sigma}\right)
\end{array}\right)
$$

where $\Theta_{\sigma}$ and $\chi_{\sigma}$ are complex functions; the function $\chi_{\sigma}$ does not contribute to the conductance (see Eq. (8) below ). Equations (3) and (4) are linear due to the smallness of the amplitude for magnetic spin-flip scattering, $\left|r_{s f}\right| \ll 1$, and are valid in the temperature interval $k_{B} T \ll \Delta$ which includes the Thouless enegy $k_{B} T_{T h}=\hbar D / L^{2} \ll \Delta$.

In order to calculate the spin-flip amplitude $r_{s f}$ one needs to know the detailed character of the magnetic inhomogeneity. A quantitative theory can only be formulated in case the magnetic structure is known in the experiment of interest. In the absence of any precise information about the magnetic structure of the samples used in existing experiments, we turn to illustrative examples of magnetic disorder and restrict ourselves to making only qualitative comparisons with experiments. We will consider two such examples: (i) the spin-splitting magnetic scattering is due to a multi-domain structure with non-colinear magnetization in the neihgbouring domains and (ii) colinear magnetization of neighboring domains but with spin-splitting scattering in the domain wall.

In case (i) Rabi oscillations of the spin direction appear, and by solving the Eilenberger equation one can show the probability amplitude $r_{s p}$ to be

$$
\left.r_{s f}=\left(h_{x}^{(0)}+i h_{y}^{(0)}\right) / I_{0}\right) \sin \left(\frac{I_{0} L_{D}}{\hbar v_{F}\left|n_{x}\right|}\right) .
$$

Here $\mathbf{h}^{(\mathbf{0})}$ is the magnetic moment in the domain closest to the superconductor, $L_{D}$ is the width of the domain (see Fig.1). Equation (6) is valid if the component of the electron/hole momentum perpendicular to the interface between the domains is not too small: $n_{x}^{2} \gg I_{0} / \epsilon_{F}$; we

that $\left\langle n_{x} \hat{\mathbf{g}}\right\rangle$ does not depend on $x$ ( $\left.15 \|\right)$ and hence connects solutions of the Eilenberger equation in the ballistic region $|x| \ll l_{0}$ with solutions of the Usadel equation in the diffusive region. 
have neglected the contribution of the domain wall itself by appealing to the smallness of its width $L_{d w}, L_{d w} \ll$ $L_{D}$.

In case (ii) we have $h_{x}^{(0)}=h_{y}^{(0)}=0$, there are no Rabi oscillations (see Eq. (6)) and only the domain wall "split" the spin, provided that $L_{d w}$ is of the order or less than $\xi_{I_{0}}=\hbar v_{F} / I_{0}$. For the case $L_{d w} \ll \hbar v_{F}\left|n_{x}\right| / I_{0}$ the probability amplitude for splitting caused by the domain wall is

$$
r_{s f}=\frac{L_{d w} I_{0}}{\hbar v_{F}\left|n_{x}\right|} .
$$

In order to calculate the conductance we follow Ref. [1] and find that the excess conductance can be written as

$$
\frac{\delta G}{G_{N}}=-\frac{1}{16 T} \sum_{\sigma=-1}^{1} \int_{-\infty}^{\infty} d \epsilon \frac{\partial f_{0}}{\partial \epsilon}\left(\frac{1}{L} \int_{0}^{L} d x\left(\operatorname{Re} \Theta_{\sigma}\right)^{2}\right) .
$$

The solution of the Usadel equation (3) with the boundary condition given by Eq.(4) at $x=0$ and by $\Theta_{\sigma}=0$ at $x=L$, is

$$
\Theta_{\sigma}=\sigma \frac{v_{F}\left|\left\langle\left|n_{x}\right| r_{s f}\right\rangle\right|}{D k(\epsilon)} \frac{\sinh (k(\epsilon)(x-L))}{\cosh (k(\epsilon) L)},
$$

where $k(\epsilon)=(1+i) \sqrt{\epsilon / \hbar D}$.

Equation (9) shows that the superconducting correlations due to the spin-splitting processes in the magnetic inhomogeneous region decay exponentially in the ferromagnet and vanish at distances of order $L_{T}$ (for energies $\epsilon \sim k T)$ corresponding to the superconducting correlation length in non-magnetic materials.

Inserting Eq. (9) into Eq. (8) one obtains an excess conductance that can be expressed as

$$
\delta G / G_{0}=\gamma f\left(T / T_{T h}\right)
$$

where

$$
\gamma=\left|\left\langle\left|n_{x}\right| r_{s f}\right\rangle\right|^{2}\left(L / l_{0}\right)^{2}
$$

and $f\left(T / T_{T h}\right)$ is a dimensionless function, the temperature dependence of which is presented in Fig.2,

$$
\begin{gathered}
f(x)=\frac{1}{x} \int_{0}^{\infty} d t \cosh ^{-2}\left(t^{2} / 2 x\right) \\
\times\left(\operatorname{Re} \frac{\sinh (2(1+i) t)-2(1+i) t}{4(i-1) t^{2} \cosh ^{2}([(1+i) t])}+\frac{\sinh 2 t-\sin 2 t}{4 t^{2}|\cosh (1+i) t|^{2}}\right)
\end{gathered}
$$

Using experimental values of the parameters taken from Ref. [6] $D=10 \mathrm{~cm}^{2} / \mathrm{s}$ and $T / T_{T h}=50$, and with the reasonable assumption that $r_{s f} \sim 10^{-1}$ our result for the excess resistance $\delta R \approx-10 \mathrm{Ohm}$ is agreement with the experiment. The temperature dependence of the excess conductance in the range $T \sim T_{T h}$ is shown in Fig.2.
For higher temperatures, $T \sim \Delta / k_{B} \gg E_{T h}$ our theory is not valid and contributions of order $k T / \Delta \sim 1$ can modify the temperature dependence of the resistance. Additional measurements around the Thouless temperature (where the proximity effect is most pronounced) would permit a comparison with the temperature dependence coming from the long-range proximity effect described by our theory. However, additional investigations of the magnetic structure of the $\mathrm{F} / \mathrm{S}$ interface are needed to carry out a complete comparison with the theory. Multidomain ferromagnets suitable for these studies can be created in various ways. It was recently demonstrated 17] that grain boundaries, magnetic inhomogeneities (including non-colinear magnetic domains) can be introduced in a predetermined position in a ferromagnet film by controlling the epitaxial growth. Experiments, where such magnetic inhomogeneities are intentionally created, would permit long-range proximity effects to be studied in ferromagnet-superconductor structures.

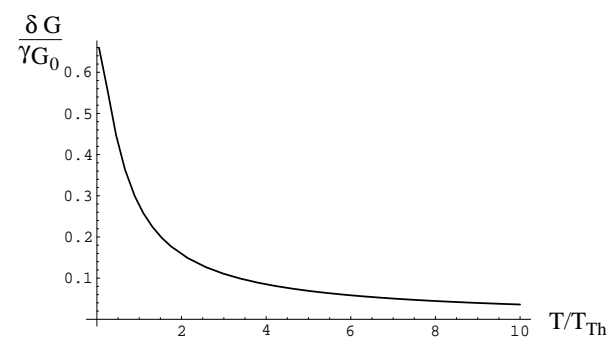

FIG. 2. Temperature dependence of the normalized excess conductance (see Eqs.(10) and (11)).

In conclusion, we have shown that spin-splitting scattering related to magnetic inhomogeneities modifies the sin-selection rule governing Andreev reflections at a ferromagnetic normal metal - superconductor interface. As a result a long-range proximity effect, due to correlations between spin-aligned electrons and holes, appears (a spin triplet proximity effect). Estimations of the value of the excess conductance are consistent with experiments 8 .

After this work was completed we learned that a similar problem have been addressed recently 18]. Considering a somewhat different model for magnetic inhomogeneity and discussing the case of a weak proximity effect (corresponding to a low-transparency tunnel barrier at the F/S interface) the authors of Ref. [18] have come to the same conclusion as we have when it comes to the existence of a long-range triplet proximity effect in magnetically inhomogeneous ferromagnets.

We acknowledge useful discussions with E.V. Bezuglyi and Z.G. Ivanov. We are grateful to Z.G. Ivanov for calling to our attention experimental possibilites to observe 
the effect predicted by our theory.

[1] C.J. Lambert and R. Raimondi, J. Phys.: Condens. Matter, 10, 901 (1998).

[2] K. Kawaguchi and M. Sohma, Phys. Rev. B46, 14722 (1992).

[3] R.J. Soulen et al., Science, 282, 85 (1998).

[4] A.M. Goldman, V. Vas'ko, P. Kraus, and K. Nikolaev, J. Magn. Magn. Mater., 200, 69 (1999).

[5] L. Lazar et al., Phys. Rev. B61, 3711 (2000).

[6] V.T. Petrashov, I.A. Sosnin, I. Cox, A. Parson, and C. Troadec, Phys. Rev. Lett. 83, 3281 (1999).

[7] V.T. Petrashov, I.A. Sosnin, I. Cox, A. Parson, and C. Troadec, Journal of Low Temp. Phys., 118, 689 (2000).

[8] M. Giroid, H. Courtois, K. Hasselbach, D. Mailly, B. Pannetier, Phys. Rev. B58, R11872 (1998).

[9] F. Zhou, B. Spivak, cond-mat/9906177.

[10] F.S. Bergeret, K.B. Efetov, and A.I. Larkin, Phys. Rev. B62, 11872 (2000).

[11] J.-X. Zhu, C.S. Ting, Phys. Rev. B61, 1456 (2000).

[12] E.A. Demler, G.B. Arnold, and M.R. Beasly, Phys. Rev. B55, 15174 (1997).

[13] G.M. Eilenberger, Z. Phys. 214, 195 (1968).

[14] K.D. Usadel, Phys. Rev. Lett. 25, 507 (1970).

[15] M. Yu. Kuprianov and V.F. Lukichev, Sov. Phys. JETP 67, 1163 (1988).

[16] W. Belzig, F. K. Wilhelm, C. Bruder, G. Schön, and A.D. Zaikin, Superlattices and Microstructures 25, 1252 (1999).

[17] R. Mathieu, P. Svedlindh, R. A. Chakalov, and Z.G. Ivanov, Phys. Rev. B62, 3333 (2000).

[18] F.S. Bergeret, A.F. Volkov, and K.B. Efetov, condmat/0011425. 


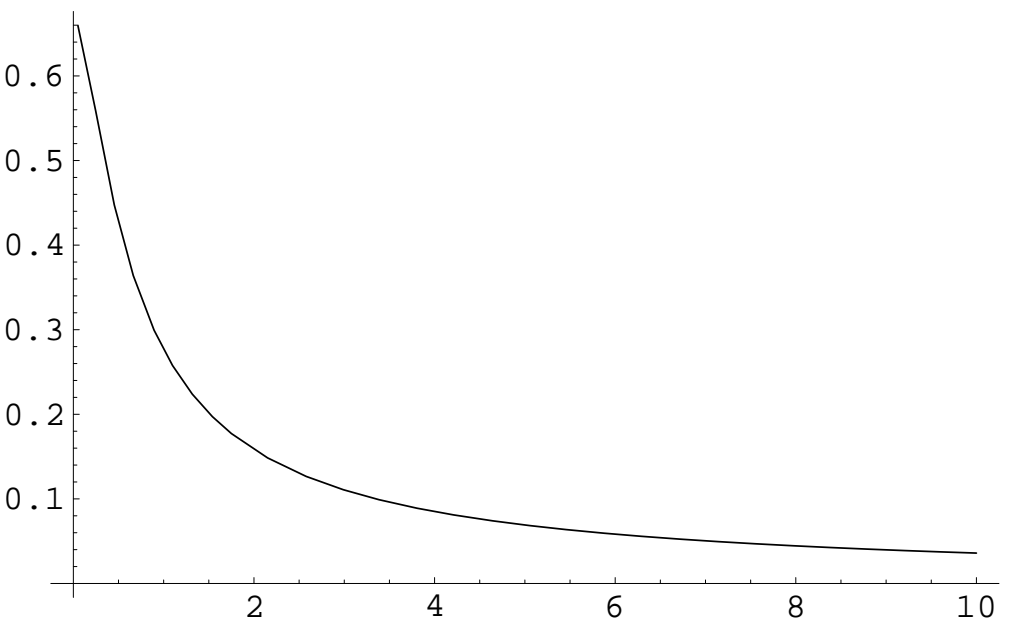

